\title{
PREDICTING TELECOMMUNICATION TOWER COSTS USING FUZZY SUBTRACTIVE CLUSTERING
}

\author{
Mohamed MARZOUK ${ }^{\mathrm{a}, \mathrm{b}}$, Mohamed ALARABY ${ }^{\mathrm{b}}$ \\ a Structural Engineering Department, Faculty of Engineering, Cairo University, Giza, Egypt \\ ${ }^{b}$ Construction Engineering and Management Program, Nile University, 12677 Giza, Egypt
}

Received 26 Jun 2012; accepted 03 Oct 2012

\begin{abstract}
This paper presents a fuzzy subtractive modelling technique to predict the weight of telecommunication towers which is used to estimate their respective costs. This is implemented through the utilization of data from previously installed telecommunication towers considering four input parameters: a) tower height; b) allowed tilt or deflection; c) antenna subjected area loading; and d) wind load. Telecommunication towers are classified according to designated code (TIA-222-F and TIA-222-G standards) and structures type (Self-Supporting Tower (SST) and Roof Top (RT)). As such, four fuzzy subtractive models are developed to represent the four classes. To build the fuzzy models, $90 \%$ of data are utilized and fed to Matlab software as training data. The remaining 10\% of the data are utilized to test model performance. Sugeno-Type first order is used to optimize model performance in predicting tower weights. Errors are estimated using Mean Absolute Percentage Error (MAPE) and Root Mean Square Error (RMSE) for both training and testing data sets. Sensitivity analysis is carried to validate the model and observe the effect of clusters' radius on models performance.
\end{abstract}

Keywords: cost estimating, quantity takeoff, telecommunication towers, fuzzy subtractive modelling, sensitivity analysis.

\section{Introduction}

Modelling in construction proved its effect on predicting data. Many models were developed with some mathematical operations to guide practitioners with the expected scenario that most likely take place during construction phase. Theoretical studies have shown that fuzzy models are capable to approximate complex functions on an easy to use model at acceptable degree of accuracy. As such, the performance of the imitated system can be studied. One of the main challenges in modelling is data availability which aids in better initiation of the model and minimizing the total errors of estimation. Cost estimating process is repetitive and performed throughout all phases (conceptual, schematic, design development, and procurement) of the project. The accuracy of cost estimating depends on availability of information and time allowance to carry out the estimate (Gould 1997). Conceptual cost estimate is associated with limited information about the project and is used as a basis for comparison with future cost estimates as well. A number of modelling techniques have been developed in construction to provide conceptual cost estimate (Ji et al. 2012; Jin et al. 2012; Fragkakis et al. 2011; El Asmar et al. 2011; Marzouk, Ahmed 2011; Tatari, Kucukvar 2011; Yu, Skibniewski 2010; Cheng et al. 2009; Chou 2009; Wang et al. 2008; Kim et al. 2004; Trost, Oberlender 2003; Siqueira
1998; Hegazy, Ayed 1998; Creese, Li 1995) for different construction projects such as highways, bridges, and low-rise structural steel buildings. These modelling techniques utilize several approaches including multivariate regression (Fragkakis et al. 2011; Trost, Oberlender 2003), neural networks (Yu, Skibniewski 2010; Cheng et al. 2009; Siqueira 1998; Hegazy, Ayed 1998; Creese, Li 1995), and Case-Based Reasoning (Jin et al. 2012; Ji et al. 2012; Marzouk, Ahmed 2011; Chou 2009; Wang et al. 2008).

Zadeh (1965) introduced fuzzy set theory to model uncertainty in human cognitive process. Fuzzy research has been applied in construction management to address two broad fields, including: fuzzy set/fuzzy logic; and hybrid fuzzy techniques. Fuzzy research has four main categories including (Chan et al. 2009): decision making; performance; evaluation/assessment; and modelling. Applications of fuzzy set and fuzzy logic include (but are not limited to) contractor selection (Singh, Tong 2005), sustainable residential buildings (Seo et al. 2004), and site layout planning (Tam et al. 2002). Marzouk (2005) presented a fuzzy clustering model for estimating the budget cost of academic buildings in Egypt. Ten parameters influencing the cost of academic buildings were identified. Four of them have been elected to be significant via step-wise regression. Marzouk and

Corresponding author: Mohamed Marzouk

E-mail:mm_marzouk@yahoo.com 
Moselhi (2004) presented a two-step fuzzy clustering method for estimating haulers' travel time. The estimated travel time takes into account the acceleration and deceleration in the transition zones. The developed method utilizes linear regression and fuzzy subtractive clustering. Seven factors influencing haulers' travel time were first identified and their significance was then quantified using linear regression. Clustering algorithms suit data compression and construction model and are wildly used to manage and classify data (Sastria et al. 2008). The main function of clustering is to gather sets of similar given objectives into groups of the same class that has common features. A cluster algorithm technique should have some properties to initiate a model with satisfying results. These include (Suryavanshi et al. 2005): a) stability in generating clusters, meaning that the clusters cannot be significantly altered; b) robustness of the model which means minor errors in sorting the objectives lead to few changes in the clustering; c) order insensitivity which means clusters composition is not impacted with the order in which objects are processed; and d) maintainability which means maintaining of clusters should be practical and efficient.

This study focuses on the subtracting clustering method (Chiu 1994), as it is an advanced technique established on mountain clustering method. To avoid being confronted with the risk of over fitting the data in case of large number of rules, suitable cluster radius has to be chosen. Sugeno-Type first order is utilized to represent the behaviour of fuzzy models in order to optimize model performance. A total of 586 previously erected telecommunication towers are classified into four classes (F_SST, F_RT, G_SST, and G_RT) to represent their categories with respect to designated code (TIA-222-F and TIA-222-G standards) and structures type (Self-Supporting Tower (SST) and Roof Top (RT)). Each class is divided into training and testing data with different cluster radius, from 0.2 till 0.65 with 0.05 intervals, and the results were recorded. The results revealed that the developed fuzzy subtractive models are effective modelling tool in providing the takeoff of telecommunication towers. To validate the developed fuzzy subtractive models, two types of errors Absolute Percentage Error (MAPE) and Root Mean Square Error (RMSE) are estimated for both training and testing data results. Result graphs are drawn to provide a comparison and detect models' performance at different clusters' radiuses. The subtractive models aid cost estimators (either from owners' side or contractors' side) who are working in telecommunication towers field to have an idea about the cost of their project. It is worth noting that the cost of civil work scope in telecommunication towers might reach up to $70 \%$ of the total cost, representing a sizable portion. Therefore, the proposed subtractive models act as a decision support tool that can be used to predict the costs of telecommunication towers projects at acceptable accuracy level once their specifications are set.

\section{Background review}

In this study, telecommunication towers are classified according to designated code and structure type. Below sub-sections provide background review for the different classes of telecommunication towers.

\subsection{Telecommunication towers design standards}

Telecommunications Industry Association (TIA) is responsible for providing a structural standard for antenna supporting structure. The main goal of the TIA is to serve the public interests through eliminating misunderstandings between manufacturers and purchasers, facilitating interchangeability and improvement of products for their particular needs. Besides this, standards provide recognized work for antenna supporting structure to minimize the design loads, giving design criteria for these structures. Both TIA-222-F and TIA-222-G are used as different standards for telecommunication towers. TIA-222-F was first published in 1996, this standard was used during the time where most nations were building telecommunication towers at the start time of mobile technology (TIA/EIA-222-F). While TIA-222-G was first published in 2006 (ANSI/TIA-222-G 2005). It calculates wind speed in a different manner, compared to TIA-222-F. The basic wind speed (according to TIA222-F) is the fastest wind speed which leads to the most worst-case wind speed maintained for a linear meter of air. While most wind gauges do not measure the fastest wind speed this way. Considering this fact, TIA-222-F standard leads to heavy structure according to fixed constant in the wind load equation and it is not preferred from the economic point of view. In TIA-222-G standard, the 3-second gust for its definition of wind speed is adopted and a conversion formula is used to translate the fastest meter to a 3 -second gust. The 3 -second gust wind speed is the highest sustained gust over a 3-second period of time. Further, TIA-222-G standard divides structures into three exposure categories (B, C \& D) and it gives the wind load equation for each category. Exposure category $B$ represents urban and suburban areas, wooded areas, or other terrain with numerous closely spaced obstructions having the size of single-family dwellings or larger. Exposure category $\mathrm{C}$ represents open terrain with scattered obstructions having heights generally less than $30 \mathrm{ft}$. Whereas, Exposure category D represents unobstructed shorelines exposed to wind flowing over open water (excluding shorelines in hurricane prone regions) for a distance of at least 1 mile. As such, TIA-222-G standard provides more flexibility and economical consideration to the designer.

\subsection{Structure types of telecommunication towers}

Telecommunication Towers are classified, according to structure types, to Self-Supporting Tower (SST) and Roof Top (RT). SSTs are either square or triangular tower with three or four legs. They are made of galvanized angular or tubular elements, designed on a base pattern. 
SSTs can stand without any wires to reduce tension as in the mast design. Their height ranges from $20 \mathrm{~m}$ to $160 \mathrm{~m}$, depending on the site requirements (Figure 1a). The weight of SSTs ranges from 2.5 tons to 120 tons. SSTs are used for medium to heavy loads and they are generally suitable for primary cellular sites, MW backbone sites, or central communication hubs. On the other hand, Roof Top (RT), or stub tower, can be described as a telecom square or triangular tower made from angular or tubular elements. RTs consist of galvanized steel parts and they usually situated over buildings; roofs. Their height ranges from $9 \mathrm{~m}$ to $21 \mathrm{~m}$ (Fig. 1b). The weight of RTs ranges from 0.5 tons till 2.5 tons. Building heights provide additional height for the stubs. To construct SSTs, an area of $10 * 10 \mathrm{~m}$ is required to support construction phases of excavation, replacement, plain concrete, reinforced concrete, erection of the tower and equipment installation. Concrete base of SSTs can be either isolated footing or raft foundation or even piles (in case of clay soil sites). It takes an average of nine to twelve days to complete erection of a single SST. While it takes one or two days to erect a RT. The foundation of RT is galvanized steel beams which are rested on the buildings' roof columns. An area of $4 * 4 \mathrm{~m}$ is required to construct RTs towers It is worth noted that the function of the two structure types (SST and RT) is to support antenna, however, the SST needs an open area to cover signals between two sites. RT is used in crowded areas with high population, where there are no available open areas to construct SSTs.

\subsection{Fuzzy logic}

Fuzzy logic is represented in a fuzzy inference system (FIS), with the "IF-THEN" rules, and it generates the outputs from given inputs using mathematical operations on membership functions. Many rules in fuzzy logic system match inputs to some degree, and they have their impact on the outputs. The FIS enables the users to notice the relationship between input features and output classes, which otherwise cannot be observed. Zadeh (2004) pointed out that the guiding principle of fuzzy logic is to exploit the tolerance for imprecision, uncertainty, and partial truth to achieve tractability, robustness, and low cost solution. This led the fuzzy logic to have an effect over modelling and the predicting of complicated problems as well as with unverified data. Different cluster algorithms have been reviewed: 1) fuzzy C-Means (FCM) clustering method (Bezdek et al. 1992); 2) mountain clustering method (Yager, Filev 1994); and 3) subtractive clustering method (Chiu 1994). Subtractive clustering method (Chiu 1994) is an extension of the mountain clustering method, where the potential is calculated for the data rather than the grid points. Cluster analysis is the task of assigning a set of objects into groups (called clusters) so that the objects in the same cluster are more similar (in some sense or another) to each other than to those in other clusters, it's a statistic technique that is used to

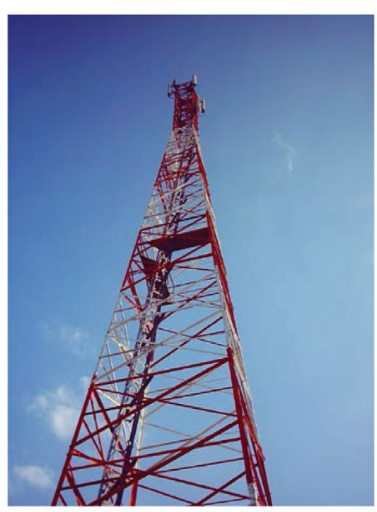

a) Self-Supporting Tower (SST)

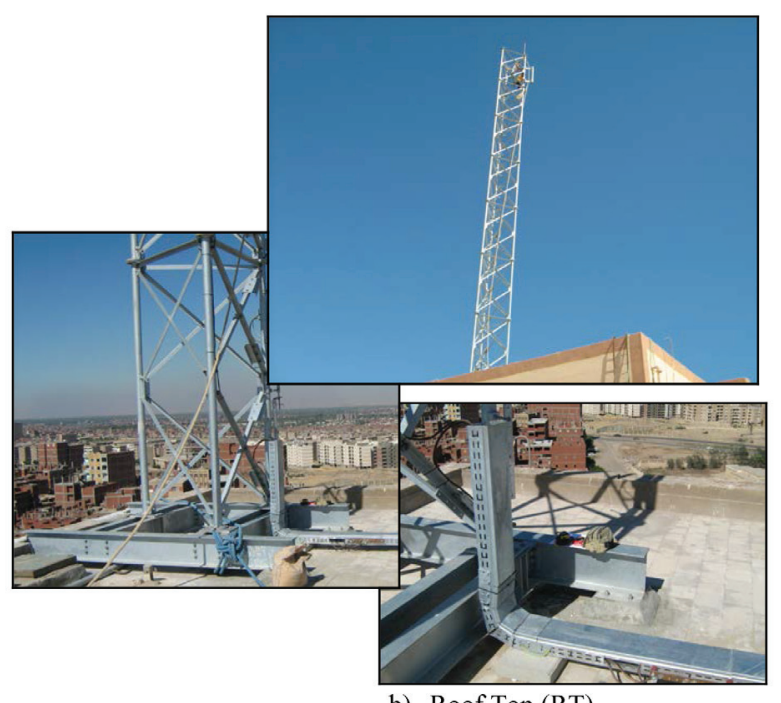

b) Roof Top (RT)

Fig. 1. Structure types of telecommunication towers

segregate groups of data. To develop fuzzy model, five steps are followed as shown in Figure 2. These steps are detailed as follows:

Step 1: Calculating the potential $\left(P_{i}\right)$ after normalization of all data points in the training data set to equalize its coordinate range in all directions. The data point, with much neighbourhood with the data points around it, will have maximum potential value than remaining data points. The first radius cluster $\left(r_{a}\right)$ is given a positive value to determine the potential of first data cluster which is a function with its distance to all the other data points:

$$
\begin{gathered}
P_{i}=\sum_{j=1}^{n} e^{-\alpha\|x i-x j\|^{2}} ; \\
\alpha=\frac{4}{r_{a}^{2}} .
\end{gathered}
$$

Step 2: The highest potential $P_{i}^{*}$ will be set as the first cluster of the model, then revise the potential for each data points by Eqns (3) and (4). Chiu (1994) advised to consider $r_{b}$ equals $1.5 r_{a}$ to avoid new cluster radius to be near the first born cluster: 


$$
\begin{gathered}
P_{i}=P_{i}-P_{1}^{*} e^{-\beta\|x i-x j\|^{2}} \\
\beta=\frac{4}{r_{b}^{2}} .
\end{gathered}
$$

Step 3: Repeat the previous step for all remaining clusters by a subtracting process, where each time new cluster found, the data points potential have been revised and the data points near the defined cluster radius will get the most reduced potential and of course it most likely will not to be chosen in the next cluster. Eqn (5) is used to choose the next cluster, and then the clustering process will be accepted if the Eqn (6) is applicable:

$$
\begin{gathered}
P_{i}=P_{i}-P_{k}^{*} e^{-\beta\|x i-x j\|^{2}} ; \\
\frac{d_{\min }}{r_{a}}+\frac{P_{k}^{*}}{P_{1}^{*}} \geq 1 .
\end{gathered}
$$

Step 4: after determining all the clusters, considering every cluster centre has its rule "membership value" that can describe system behaviour, and with putting $y$ as an input vector, the output vector $(z)$ is calculated using Eqns (7) and (8):

$$
\begin{gathered}
\mu_{i}=e^{-\alpha\left\|y-y_{i}^{*}\right\|^{2}} ; \\
z=\frac{\sum_{i=1}^{c} \mu_{i} z_{i}^{*}}{\sum_{i=1}^{c} \mu_{i}} .
\end{gathered}
$$

where: $\mu_{i}$ is the membership value of cluster $i$; and $\alpha$ is constant as per Eqn (2).

Step 5: Least-Square optimization Takagi-Sugeno used as polynomial function of first order. Supposing a linear output for each cluster $\left(z^{*}=G_{i} y+h_{i}\right)$, optimization is performed to minimize the square error shown in Eqns (9), (10) and (11):

$$
\begin{gathered}
z=\sum_{j=1}^{c} \rho_{i}\left(G_{i} y+h_{i}\right) \\
\rho_{i}=\frac{\mu_{i}}{\sum_{j=1}^{c} \mu_{i}}
\end{gathered}
$$

$$
\left[\begin{array}{c}
z_{1}^{T} \\
\vdots \\
z_{n}^{T}
\end{array}\right]=\left[\begin{array}{ccccc}
\rho_{1,1} y_{1}^{T} & \rho_{1,1} & \cdots & \rho_{c, 1} y_{1}^{T} & \rho_{c, 1} \\
& & \vdots & & \\
\rho_{1, n} y_{1}^{T} & \rho_{1, n} & \cdots & \rho_{c, n} y_{c}^{T} & \rho_{c, n}
\end{array}\right]\left[\begin{array}{c}
G_{1}^{T} \\
h_{1}^{T} \\
\vdots \\
G_{c}^{T} \\
h_{c 1}^{T}
\end{array}\right] .
$$

\section{Research methodology}

\subsection{Data collection}

In this study, four inputs parameters are considered which are: antenna subjected loading, tilt, height and wind speed. The predicted output is the expected weight of the telecommunication tower. The towers' data is taken from a library that has the records of previously erected towers with their respective inputs and output. Such data are used to develop fuzzy subtractive models. Data are divided into four groups as follows:

- Group 1: F_SST contains 250 SST towers' records, designed using F Standard, that are divided into two sets: 225 records for training and 25 records for testing.

- Group 2: F_RT contains 43 RT towers' records, designed using $\mathrm{F}$ Standard, that are divided into two sets: 38 records for training and 5 records for testing.

- Group 3: G_SST contains 235 SST towers' records, designed using $\mathrm{G}$ Standard, that are divided into two sets: 212 records for training and 24 records for testing.

- Group 4: G_RT contains 40 RT towers' records, designed using $\mathrm{G}$ Standard, that are divided into two sets: 36 records for training and 4 records for testing.

\subsection{Model implementation}

The proposed models are capable to estimate the cost of the towers using the predicted weight using Eqn (12). Figure 3 illustrates schematic diagram of models' inputs and output which are implemented using Matlab software:

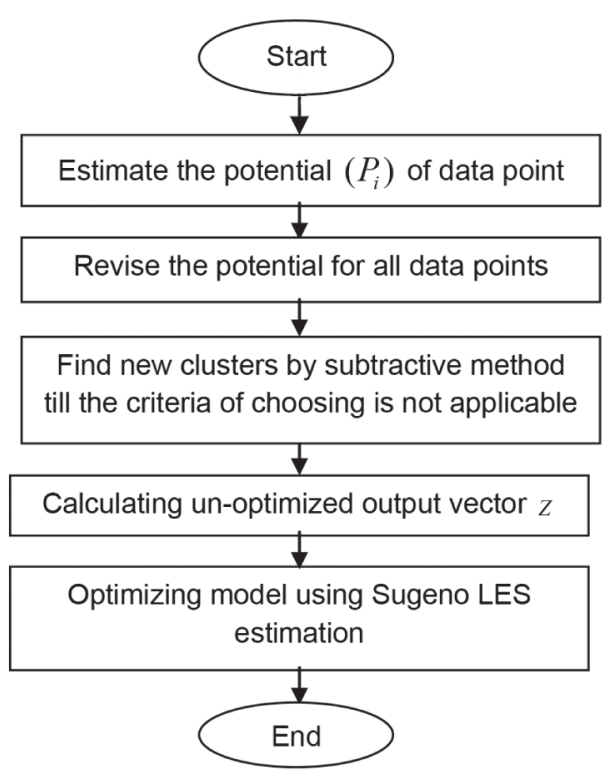

Fig. 2. Fuzzy clustering subtractive algorithm 


$$
\text { Cost }=W_{P} \times C_{T},
$$

where: $W_{p}$ is predicted weight by model; and $C_{T}$ is Cost per ton.

\subsection{Models cluster centres}

Finding clusters' centres are deemed important to start fuzzy rules and, then, optimizing the rules. The first step in finding clusters' centres is to choose data points with high potential to be selected as the clusters' centres in a group of ( $n$ )input-output data. Every single data point has its potential, which is the distance between data points and the assigned radius of clusters (ra). The potential is expressed by an exponential function stating neighbourhood. After the potentials of all data point are initialized, the potentials are revised and checked, and then the numbers of clusters are set. As a cluster centre is evaluated and data point is accepted as a cluster centre, then firing the fuzzy rules, which is accomplished by optimization is carried out. Chiu (1994) noticed that the cluster radius must suit the data points to avoid over-fitting that appears when using a small radius, however, choosing a large radius will lead to fewer clusters which then create unsatisfied model results, therefore, while choosing the cluster radius, it must fit the data points. This disadvantage of this method is the creation of a membership function with many trials in order to find a best initial set-up. However, there is also an advantage which is taking the shortest way without waiting the training data iteration to complete the system. Because of the importance of the radius ra of the clusters in Chiu method, more than one radius was used with this system to predict the results. Table 1 lists the number of clusters for the different assigned radius ra.

\subsection{Estimating models errors}

To validate fuzzy models, Mean Absolute Percentage Error (MAPE) and Root Mean Square Error (RMSE) are estimated for training and testing data sets for the four fuzzy subtractive models using Eqns (13) and (14):

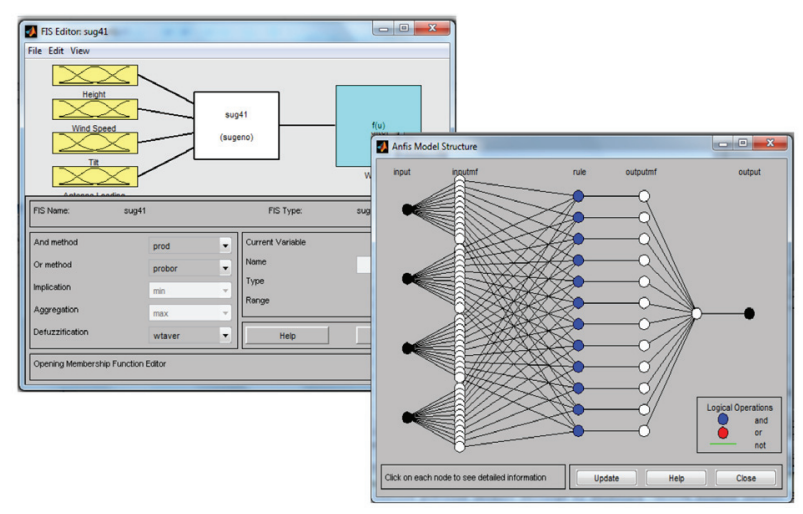

Fig. 3. Inputs and outputs of fuzzy clustering models
Table 1. Number of clusters for different assigned radius

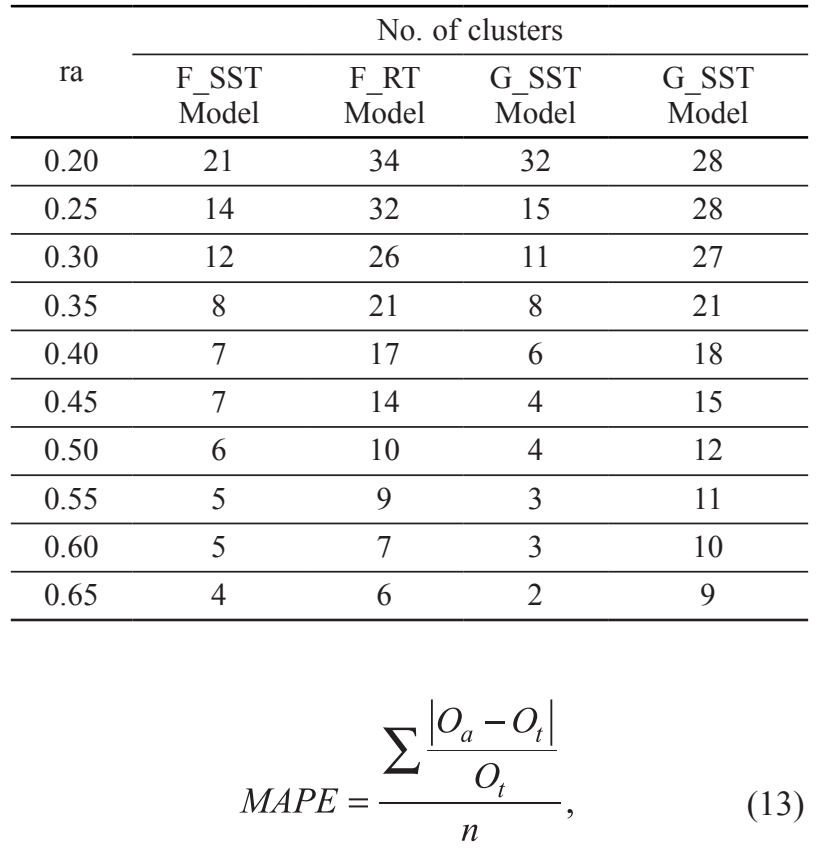

where: $n$ is the number of input of the data; $O_{a}$ is actual input of the data; and $O_{t}$ is target output from the system.

$$
R M S E=\sqrt{\frac{\sum\left(Z_{a}-Z_{t}\right)^{2}}{n}},
$$

where: $n$ is the number of input of the data; $Z_{a}$ is actual input of the data; and $Z_{t}$ is normalized target output from the system.

\section{Sensitivity analysis}

Sensitivity analysis investigates the output of mathematical model robustness and is useful for decision making support by testing the results of a model. Modellers refer to these types of models and use their results to take the right decisions, make recommendations, increase the ability to understand all the variables of the model such as inputs and outputs and the relationship between them, and understanding how the model behaves. Sensitivity analysis leads to improving final solutions and improving implementation. Sensitivity analysis is carried out on the developed fuzzy clustering models by considering different values for the assigned radius of clusters $\left(r_{a}\right)$ and tracing the output values. Figures 4 and 5 depict the values for Mean Absolute Percentage Error (MAPE) and Root Mean Square Error (RMSE) for the four fuzzy clustering models. For Roof Top (RT) models $\left(F_{-} R T\right.$ and $G_{-} R T$ ), the values of Mean Absolute Percentage Error (MAPE) and Root Mean Square Error (RMSE) are close to zero as per as per Figure $4 b$ and $d$ and Figure $5 b$ and $\mathrm{d}$, respectively. This is attributed to the fact that the welltrained model due to the limited number of telecommunication towers types available in training data sets. The following results have been obtained from the sensitivity analysis for testing data sets (Table 2): 


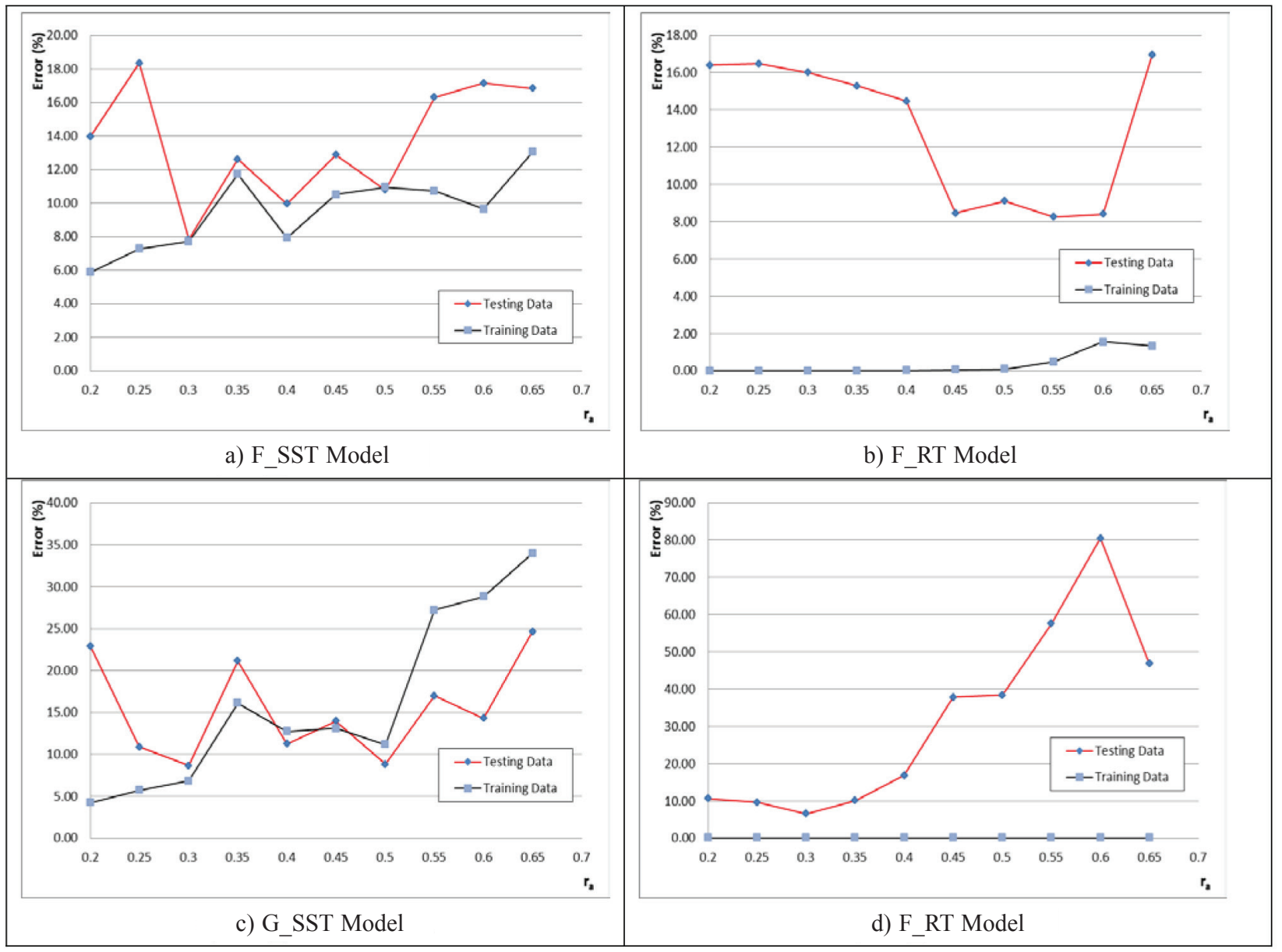

Fig. 4. Models' mean absolute percentage error (MAPE)

- For F_SST Model, both MAPE and RMSE have minimum values when ra equals 0.3 .

- For F_RT Model; both MAPE and RMSE have minimum values when ra equals 0.55 .

- For G_SST Model, MAPE has minimum value when ra equals 0.3 , whereas, RMSE is minimum when ra equals 0.5 .

- For G_RT Model, both MAPE and RMSE have minimum values when ra equals 0.3 .

Table 2. Sensitivity analysis results

\begin{tabular}{lcccc}
\hline \multirow{2}{*}{ Model } & \multicolumn{2}{c}{ MAPE } & \multicolumn{2}{c}{ RMSE } \\
\cline { 2 - 5 } & ra & Error (\%) & ra & Error (\%) \\
\hline F_SST & 0.3 & 7.83 & 0.3 & 10.30 \\
\hline F_RT & 0.55 & 8.25 & 0.55 & 9.06 \\
\hline G_SST & 0.3 & 8.65 & 0.5 & 10.74 \\
\hline G_RT & 0.3 & 6.62 & 0.3 & 7.73 \\
\hline
\end{tabular}

\section{Conclusions}

This paper presented a fuzzy subtractive modelling technique to predict the weight of telecommunication towers which is used to estimate their respective costs. Four input parameters are considered in the modelling: tower height, allowed tilt or deflection, antenna subjected area loading, and wind load. Four fuzzy subtractive models were developed to take into consideration telecommunication designated code (TIA-222-F and TIA-222-G standards) and structures type (Self-Supporting Tower (SST) and Roof Top (RT)). These models are F_SST, F_RT, G_SST, and G_RT. Towers' data were taken from a library that has the records of previously erected 568 towers with their respective inputs and output. Four inputs parameters were considered in the developed fuzzy subtractive models which are; antenna subjected loading, tilt, height and wind speed. The towers' data were classified into training data $(90 \%)$ and testing data $(10 \%)$. To validate the fuzzy model, Mean Absolute Percentage Error (MAPE) and Root Mean Square Error (RMSE) were estimated for training and testing data sets for the four fuzzy subtractive models. Sensitivity analysis was carried out on the developed fuzzy clustering models by 


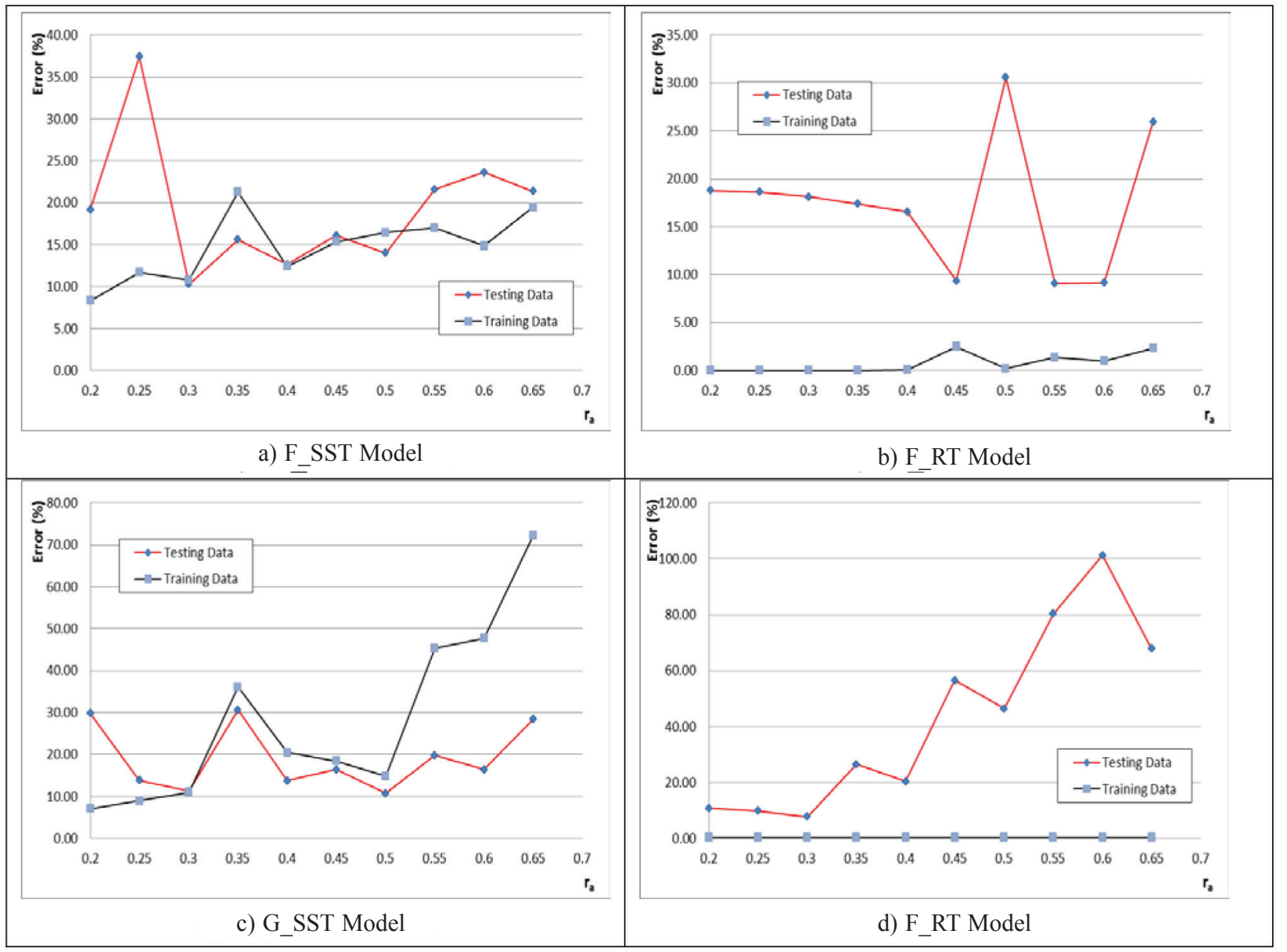

Fig. 5. Models' root mean square error (RMSE)

considering different values for the assigned radius of clusters (ra) that range from 0.2 to 0.65 . Models' output values were traced to identify minimum Mean Absolute Percentage Error (MAPE) and Root Mean Square Error (RMSE) for each model. It was found that MAPE and RMSE have minimum values for the same radius of cluster for F_SST, F_RT and G_RT Models. Whereas, the minimum values of MAPE and RMSE are obtained at different radius of clusters for G_SST model.

\section{References}

ANSI/TIA-222-G. 2005. Structural Standard for Antenna Supporting Structures and Antennas, Telecommunications Industry Association.

Bezdek, J. C.; Hathaway, R. J.; Sabin, M. J.; Tucker, W. T. 1992. Convergence theory for fuzzy C-means: counter examples and repairs, in Bezdek, J. C.; Pal, S. K. (Eds.). Fuzzy Models for Pattern Recognition. New York: IEEE Press, 138-142.

Chan, A. P. C.; Chan, D. W. M.; Yeung, J. F. Y. 2009. Overview of the application of "fuzzy techniques" in construction management research, Journal of Construction Engineering and Management ASCE 135(11): 1241-1252. http://dx.doi.org/10.1061/(ASCE)CO.1943-7862.0000099

Cheng, M.-Y.; Tsai, H.-C.; Hsieh, W.-S. 2009. Web-based conceptual cost estimates for construction projects using
Evolutionary Fuzzy Neural Inference Model, Automation in Construction 18(2): 164-172.

http://dx.doi.org/10.1016/j.autcon.2008.07.001

Chiu, S. L. 1994. Fuzzy model identification based on cluster estimation, Journal of Intelligent and Fuzzy Systems 2: 267-278.

Chou, J.-S. 2009. Web-based CBR system applied to early cost budgeting for pavement maintenance project, $E x$ pert Systems with Applications 36(2) Part 2: 2947-2960.

Creese, R. C.; Li, I. 1995. Cost estimating of timber bridges using neural networks, Cost Engineering 73(5): 17-22.

El Asmar, M.; Hanna, A. S.; Whited, G. C. 2011. New approach to developing conceptual cost estimates for highway projects, Journal of Construction Engineering and Management 137(11): 942-949.

http://dx.doi.org/10.1061/(ASCE)CO.1943-7862.0000355

Fragkakis, N.; Lambropoulos, S.; Tsiambaos, G. 2011. Parametric model for conceptual cost estimation of concrete bridge foundations, Journal of Infrastructure Systems 17(2): 66-74.

http://dx.doi.org/10.1061/(ASCE)IS.1943-555X.0000044

Gould, F. E. 1997. Managing the construction process. New Jersey: Prentice-Hall, Inc. 338 p.

Hegazy, T.; Ayed, A. 1998. A neural network model for parametric cost estimation of highway projects, Journal of Construction Engineering and Management ASCE 24(3): 210-218.

http://dx.doi.org/10.1061/(ASCE)0733-9364(1998)124:3(210) 
Ji, S.-H.; Park, M.; Lee, H.-S. 2012. Case adaptation method of case-based reasoning for construction cost estimation in Korea, Journal of Construction Engineering and Management 137(1): 43-52. http://dx.doi.org/10.1061/(ASCE)CO.1943-7862.0000409

Jin, R. Z.; Cho, K. M.; Hyun, C. T; Son, M. J. 2012. MRAbased revised CBR model for cost prediction in the early stage of construction projects, Expert Systems with Applications 39(5): 5214-5222. http://dx.doi.org/10.1016/j.eswa.2011.11.018

Kim, G.-H.; An, S.-H.; Kang, K.-I. 2004. Comparison of construction cost estimating models based on regression analysis, neural networks, and case-based reasoning, Building and Environment 39(10): 1235-1242. http://dx.doi.org/10.1016/j.buildenv.2004.02.013

Marzouk, M. 2005. Cost estimating of academic buildings using fuzzy clustering technique, in $6^{\text {th }}$ Construction Specialty Conference, 2-4 June 2005, Toronto, Ontario, Canada. 8 p.

Marzouk, M.; Moselhi, O. 2004. Fuzzy clustering model for estimating Haulers' travel time, Journal of Construction Engineering and Management ASCE 130(6): $878-886$.

http://dx.doi.org/10.1061/(ASCE)0733-9364(2004)130:6(878)

Marzouk, M. M.; Ahmed, R. M. 2011. A case-based reasoning approach for estimating the costs of pump station projects, Journal of Advanced Research 2(4): 289-295. http://dx.doi.org/10.1016/j.jare.2011.01.007

Sastria, G.; Liong, C.-Y.; Hashim, I. 2008. Application of Fuzzy Subtractive Clustering for Enzymes Classification, in ACC'08 Proceedings of the WSEAS International Conference on Applied Computing Conference, 27-30 May 2008, Istanbul, Turkey, 304-309.

Seo, S.; Aramaki, T.; Hwang, Y.; Hanaki, K. 2004. Fuzzy decision making tool for environmental sustainable buildings, Journal of Construction Engineering and Management ASCE 130(3): 415-423.

http://dx.doi.org/10.1061/(ASCE)0733-9364(2004)130:3(415)

Singh, D.; Tong, R. L. K. 2005. A fuzzy decision framework for contractor selection, Journal of Construction Engineering and Management 131(1): 62-69.

http://dx.doi.org/10.1061/(ASCE)0733-9364(2005)131:1(62)
Siqueira, I. 1998. Automated cost estimating system using neural networks, Project Management Journal, March 1999, $11-18$.

Suryavanshi, B. S.; Shiri, N.; Mudur, S. P. 2005. A fuzzy hybrid collaborative filtering technique for web personalization, Proceedings of $3^{\text {rd }}$ Workshop Intelligent Techniques for Web Personalization (ITWP'05), 1 August 2005, Edinburgh, Scotland.

Tam, C. M.; Tong, T. K. L.; Leung, A. W. T.; Chiu, G. W. C. 2002. Site layout planning using nonstructural fuzzy decision support system, Journal of Construction Engineering and Management 128(3): 220-231.

http://dx.doi.org/10.1061/(ASCE)0733-364(2002)128:3(220)

Tatari, O.; Kucukvar, M. 2011. Cost premium prediction of certified green buildings: a neural network approach, Building and Environment 46(5): 1081-1086. http://dx.doi.org/10.1016/j.buildenv.2010.11.009

TIA/EIA-222-F 1996. Structural standards for steel antenna towers and antenna supporting structures, American National Standards Institute (ANSI)/Telecommunications Industry Association.

Trost, S. M.; Oberlender, G. D. 2003. Predicting accuracy of early estimates using factor analysis and multivariate regression, Journal of Construction Engineering and Management ASCE 129(2): 198-204.

http://dx.doi.org/10.1061/(ASCE)0733-9364(2003)129:2(198)

Wang, H.-J.; Chiou, C.-W.; Juan, Y.-K. 2008. Decision support model based on case-based reasoning approach for estimating the restoration budget of historical buildings, Expert Systems with Applications 35(4): 1601-1610. http://dx.doi.org/10.1016/j.eswa.2007.08.095

Yager, R. R.; Filev, D. P. 1994. Generation of fuzzy rules by mountain clustering, Journal of Intelligent and Fuzzy Systems 2: 209-219.

Yu, W.; Skibniewski, M. J. 2010. Integrating neurofuzzy system with conceptual cost estimation to discover cost-related knowledge from residential construction projects, Journal of Computing in Civil Engineering 24(1): 35-44.

http://dx.doi.org/10.1061/(ASCE)0887-3801(2010)24:1(35)

Zadeh, L A. 1965. Fuzzy sets, Information and Control 8(3): 338-353. http://dx.doi.org/10.1016/S0019-9958(65)90241-X

Zadeh, L. A. 2004. Foreword to Fuzzy Logic Toolbox User's Guide. Natick: The MathWorks Inc., 3-4.

Mohamed MARZOUK. He is a Professor of Construction Engineering and Management at Department of Structural Engineering, Faculty of Engineering, Cairo University. He received his BSc and MSc in Civil Engineering from Cairo University in 1995 and 1997, respectively. He received his $\mathrm{PhD}$ form Concordia University in 2002. His research interest includes computer simulation, optimization of construction processes, fuzzy logic and its applications in construction, risk analysis, sustainability, building information modeling, and decision analysis.

Mohamed ALARABY is a Project Manager at Al Babtain LeBlanc Egypt for Telecom Engineering, Giza, Egypt. He received his BSc in structural engineering from Zagazig University in 2004 and MSc from Cairo University in 2013. His research interest includes computer simulation, Fuzzy Clustering, decision support systems, construction technology, automation in construction, and projects planning for telecommunication steel towers. 